\title{
Contribuições dos experimentos de Física em feiras e planetário itinerantes na desmistificação do aprendizado: Um estudo de caso de um grupo de educação tutorial na Unifei
}

\author{
Contributions of experiments in fairs and with an itinerant planetarium to the demythification in \\ learning Physics: A case study based on the activities of a tutoring group at Unifei \\ Contribuciones de los experimentos de Física en ferias y planetario itinerante en la desmitificación \\ del aprendizaje: Un estudio de caso de un grupo de educación tutorial en Unifei
}

Recebido: 05/06/2021 | Revisado: 11/06/2021 | Aceito: 15/06/2021 | Publicado: 30/06/2021

\author{
Marina Aparecida Barbosa de Souza \\ ORCID: https://orcid.org/0000-0003-1465-0031 \\ Universidade Federal de Itajubá, Brasil \\ E-mail: m.apsouza06@gmail.com \\ Denize Aparecida Gualberto \\ ORCID: https://orcid.org/0000-0002-8100-7890 \\ Universidade Federal de Itajubá, Brasil \\ E-mail: denizegualberto@gmail.com \\ Lourival Fernandes Nogueira Neto \\ ORCID: https://orcid.org/0000-0002-9390-250X \\ Universidade Federal de Itajubá, Brasil \\ E-mail: lourivalnetto16@gmail.com \\ Jordania Drumond Alves Soares \\ ORCID: https://orcid.org/0000-0001-6320-1457 \\ Universidade Federal de Itajubá, Brasil \\ E-mail: jordaniadrumond@gmail.com \\ Brenda Martins Fernandes \\ ORCID: https://orcid.org/0000-0002-1854-0756 \\ Universidade Federal de Itajubá, Brasil \\ E-mail: b.martinsf4@gmail.com \\ Evandro Augusto de Morais \\ ORCID: https://orcid.org/ 0000-0001-9927-7656 \\ Universidade Federal de Itajubá, Brasil \\ E-mail: evmorais@unifei.edu.br
}

\begin{abstract}
Resumo
O projeto Ciência em Órbita vem sendo desenvolvido pelo grupo PET Conexão de Saberes: Física e Popularização da Ciência (Unifei - Campus Itabira) desde 2015. Dessa forma, o presente artigo retrata uma amostra de experimentos de Física realizada pelos integrantes do PET, tendo como objetivo, compartilhar de forma clara, dinâmica e didática os conhecimentos da Física. Sendo assim, diversos experimentos são apresentados, nos quais envolvem as diversas áreas da Física Básica, como Mecânica, Termodinâmica, Eletricidade e Óptica, tais como: Globo de Plasma, Bolha de Sabão Gigante, Gerador de Van de Graaff, Bobina de Tesla, entre outros. São explorados os conceitos de Física de forma prática; além do Planetário Itinerante, no qual é possível vivenciar a Astronomia de maneira divertida e lúdica. Os experimentos foram realizados com público livre, em que as abordagens foram adequadas, em relação a faixa etária dos participantes. A metodologia utilizada neste artigo é o estudo de caso com abordagem qualitativa, no qual engloba o desenvolvimento do projeto, as suas principais características e contribuições identificadas, tal como para os participantes quanto para a equipe. Dentre estes, destaca-se os benefícios do elo entre a universidade e a sociedade, no qual promove a disseminação do conhecimento.
\end{abstract}

Palavras-chave: PET; Feira de Ciências; Planetário; Ensino; Universidade.

\section{Abstract}

The Project Ciência em Órbita has been developed by PET/Conexão de Saberes: Física e Popularização da Ciência (Unifei - Campus Itabira) since 2015. This paper depicts a set of Physics experiments performed by the PET team with aim at sharing knowledge in a didatic, dinamic clear and clear way. The experiments explore the concepts of Physics with a practical approach, comprise several different areas such as Basic Physics, Mechanics, Thermodynamics, Electricity and Optics and include Plasma Globe, Giant Soup Bubble, Van de Graaff Generator, 
Tesla Coil, and others. Besides, an itinerant Planetarium is also used to present Astronomy in a ludic and fun perspective. The experiments were presented in free, public events, always adapting the language to the age range of the audience. The methodology adopted in this paper is of a case study with qualitative analysis and comprises the development of the project and its main characteristics and contributions for both participants: the audience and the team. Among these contributions, it is worth stressing the benefits of the bond between the Society and the University as a mean to promote knowledge.

Keywords: PET; Science Fairs; Planetarium; Education; University.

\section{Resumen}

El proyecto Ciencia en Órbita ha sido desarrollado por el grupo PET Conexión de Saberes: Física y Divulgación de la Ciencia (Unifei - Campus Itabira) desde 2015. Así, este artículo presenta una muestra de experimentos de Física realizados por miembros del PET, teniendo como objetivo, compartir el conocimiento de la Física de forma clara, dinámica y didáctica. Así, se presentan varios experimentos, que involucran las diversas áreas de la Física Básica, tales como Mecánica, Termodinámica, Electricidad y Óptica, tales como: Globo de Plasma, Burbuja de Jabón Gigante, Generador Van de Graaff, Bobina de Tesla, entre otras. Los conceptos de física se exploran de forma práctica; además del Planetario Itinerante, donde podrás experimentar la Astronomía de una manera divertida y lúdica. Los experimentos se realizaron con un público libre, en el que los enfoques fueron adecuados, en relación al grupo de edad de los participantes. La metodología utilizada en este artículo es el estudio de caso con enfoque cualitativo, que engloba el desarrollo del proyecto, sus principales características y aportes identificados, tanto para los participantes como para el equipo. Entre estos, destacan los beneficios del vínculo entre la universidad y la sociedad, en el que se promueve la difusión del conocimiento.

Palabras clave: PET; Feria de Ciencias; Planetario; Enseñanza; Universidad.

\section{Introdução}

A utilização de práticas experimentais como metodologia para aprimorar processos de ensino e aprendizagem em Física e/ou Ciências são constantemente utilizadas, com benefícios, tanto na aprendizagem quanto no aumento pelo interesse nestas disciplinas. Tais práticas demonstram forte aceitação entre professores e alunos, de modo a vivenciar a ocorrência de um fenômeno que é alvo do estudo das ciências, e que contribui para que os observadores entendam os fatores que regem o funcionamento e ao incidente do objeto de estudo (Higa \& Oliveira, 2012; Pereira \& Robaina, 2020).

Dependendo do ambiente e a forma em que os processos de ensino e aprendizagem são desenvolvidos, a educação pode ser analisada por meio de três enfoques: educação formal, não formal e informal (Langhi \& Nardi, 2009). Um termo muito comum, usado quando se pensa em ensino científico no sentido do meio acadêmico para a sociedade, é a popularização da ciência, o qual se confunde historicamente com outros termos como vulgarização, difusão e alfabetização científica (Germano \& Kulesza, 2007).

É importante relembrar este conceito, considerando-o não apenas a transposição do conhecimento científico para indivíduos que não estão inseridos na academia para, em um sentido mais amplo e bem-aceito, considerar: a compreensão do mundo em que vive, as condições para formular opiniões a partir de reflexões e condições de debater, possibilitando assim uma ação cultural libertadora (Germano \& Kulesza, 2007).

Diversas ferramentas são desenvolvidas para aprimorar os processos de ensino e aprendizagem, nas quais destacam-se principalmente a experimentação e os métodos lúdicos de ensino, muito utilizados na educação infantil. O aprender com prazer por meio de jogos, brincadeiras e estímulos promove o desenvolvimento pessoal, cultural e social, facilitando os mais variados processos de expressão, socialização, comunicação e construção do conhecimento (Arantes \& Barbosa, 2017).

As atividades que desenvolvem a popularização da ciência são essenciais para o desenvolvimento da sociedade. Duas atividades merecem destaque para o cumprimento deste papel: Feira de Ciências e a Astronomia; dependendo da forma trabalhada, ambas podem se encaixar na categoria educação não formal. As primeiras feiras escolares iniciaram-se no Brasil na década de 1960, em função da difusão de diferentes abordagens de ensino, por meio da problematização e realização de experimentos para o aprendizado (Barcelos, Jacobucci, \& Jacobucci, 2010; Higa \& Oliveira, 2012; Pereira \& Robaina, 2020). 
A iniciação a práticas científicas no início da formação dos discentes permite aos alunos desenvolver competências, sintetizar fatos e adquirir a capacidade de criticar. As Feiras de Ciências constituem em uma estratégia de grande valor formativo, cujo início, desenvolvimento e apresentação dos trabalhos envolvem grande parte da comunidade. Há a necessidade do desenvolvimento do pensar para se efetivar uma aprendizagem significativa e um julgar crítico (Germano \& Kulesza, 2011; Oaigen, Bernard, \& Souza, 2013).

O uso do Planetário Itinerante auxilia no despertar para o interesse da população para assuntos relacionados à Astronomia. Constituído por uma cúpula hemisférica interna e uma série de equipamentos nos quais buscam-se projetar imagens do universo na parte central dessa cúpula, o Planetário é um local exclusivamente elaborado com objetivo de apresentar uma simulação do céu, empenhando-se para exibi-lo o mais próximo da realidade (Everding \& Keller, 2020).

O Planetário Itinerante é capaz de exibir imagens digitais projetadas na tela da cúpula de forma contínua, apresentando alta qualidade e nitidez para a plateia. Grande parte dos Planetários apresentam por meio de dados e simulações, a capacidade de acesso em tempo real (Lantz, 2011). Segundo a Sociedade Internacional de Planetário (do inglês "International Planetarium Society" - IPS), aproximadamente 1.300 cúpulas digitais estão em funcionamento nas unidades acadêmicas espalhadas pelo mundo (Zhukova \& Bukgakova, 2019).

Em um estudo realizado por meio de questionários aplicados para 240 alunos de 6 escolas com níveis socioeconômicos distintos, comprovaram que o ensino de conceitos astronômicos dentro dos Planetários são melhores absorvidos em relação à sala de aula convencional (Türk \& Kalkan, 2015). Dessa forma, possibilita aos alunos tanto de nível fundamental quanto superior, uma vivência óptica acentuada, permitindo-se uma conversão do que é visto em um aprendizado mais relevante. Além disso, promove aos estudantes uma visão mais ampla sobre alguns conceitos aplicados (Everding \& Keller, 2020).

Portanto, entre os trabalhos desenvolvidos pelo grupo de Programa de Educação Tutorial (PET) Conexões dos Saberes em Física e Popularização da Ciência (PETFIS), estão a Feira de Ciências e Planetário, ambos itinerantes e com o objetivo de ilustrar os fenômenos físicos e astronômicos, principalmente nas áreas de Mecânica, Eletricidade, Termodinâmica, Óptica e Física Moderna e Astronomia. Desse modo, as atividades são desenvolvidas nos mais diversos ambientes, considerando a faixa etária e nível de escolaridade dos participantes.

Sendo assim, ressalta-se que estas atividades abrangem os pilares fundamentais do programa de educação tutorial, compostos pela tríade de ensino, pesquisa e extensão. O grupo vem desenvolvendo atividades com foco na metodologia de ensino que aproximem a comunidade e a universidade (Costa et al., 2020; Fernandes et al., 2020; Morais et al., 2020). Assim, contribui para o aprendizado e desenvolvimento tanto dos integrantes do grupo PETFIS, quanto dos participantes externos que interagem com os experimentos.

A extensão como uma prática organizada com métodos e objetivos, produz conhecimento para a própria instituição e auxilia na transformação das ações da população. Ademais, a comunicação quando levada para a sociedade de uma maneira didática para o público escolhido, constitui um elo entre academia e sociedade (M. F. da Silva \& Mendoza, 2020).

Neste contexto, este trabalho mostra relatos de alguns experimentos realizados nas Feiras e no Planetário Itinerantes pelo PETFIS, vinculado a Unifei Campus Itabira. Além disso, tem o objetivo de realizar uma análise relacionada às contribuições que estes projetos geram em torno tanto da equipe, quanto dos participantes dos eventos.

Dessa forma, este artigo utiliza como método de pesquisa o estudo de caso do tipo explicativo com abordagem qualitativa, no qual a estrutura segue as seguintes etapas: introdução, referencial teórico, resultados e discussões e considerações finais. 


\section{Metodologia}

\subsection{Estudo de Caso}

O estudo de caso retrata um acontecimento recente ou decorrido, sendo estruturado por intermédio de diversas bases de dados, sendo por exemplo, tanto por meio de pesquisa e análises em artigos de natureza pública ou privada, quanto por entrevistas estruturadas e observações imediatas (Freitas \& Jabbour, 2010). De cunho empírico, o estudo de caso é uma metodologia na qual busca-se verificar dentro de um cenário real uma ocorrência que normalmente é atual (Miguel, 2007).

Geralmente os estudos de caso tendem a deixar claro a razão em que as decisões foram tomadas, como foram implementadas e os resultados alcançados (Miguel, 2007). Elaborar um estudo de caso é uma tarefa que exige muito do pesquisador, pelo fato de exigir muita dedicação e tempo, sendo assim considerado algo não muito fácil. $\mathrm{O}$ estudo de caso é a metodologia ideal para compreender todo o contexto do fenômeno estudado (Freitas \& Jabbour, 2010).

Para a condução do estudo de caso, e para que os objetivos da pesquisa sejam alcançados, devem-se seguir rigidamente as técnicas e métodos. Sendo assim, o estudo pode ser dividido nas seguintes etapas: revisão da literatura, planejamento dos casos, coleta de dados, análise de dados e relatório (Miguel, 2007).

\subsection{Planejamento do Estudo de Caso}

As diretrizes seguidas do estudo de caso produzido foram da pesquisa explicativa (Miguel, 2007). O presente artigo busca relatar os procedimentos e realizar uma análise dos impactos gerados pelos experimentos aplicados pela equipe PETFIS da Unifei Campus Itabira, nas Feiras e no Planetário Itinerantes, no qual será um Estudo de caso múltiplos com abordagem qualitativa.

Os dados coletados para análise foram levantados por meio da observação participante (Miguel, 2007), sendo que os experimentos realizados, tanto nas Feiras quanto no Planetário Itinerantes, eram executados pela equipe PETFIS.

Para administração do projeto, o grupo conta sempre com um aluno coordenador, que tem como objetivos observar e repassar a necessidade de manutenção dos equipamentos, organização das apresentações e selecioná-los de acordo com a Feira e/ou Planetário a ser realizado.

Os integrantes foram os responsáveis pela aplicação dos experimentos, relatos dos procedimentos, coleta de dados e extração de informações a partir destes, para assim, realizar a análise de dados.

Os objetos de estudo do presente artigo abrangem o município de Itabira e região, localizados no estado de Minas Gerais - Brasil; para escolas e/ou exposições englobantes aos finais de semanas e/ou eventos específicos como, por exemplo, Natal, Semana Nacional da Ciência e Tecnologia e Semana das Crianças.

Os experimentos foram realizados com público livre, porém com diferentes formas de abordagens, conforme faixa etária dos participantes. As informações são mais lúdicas para o público infantil, enquanto para o público mais maduro, as informações são mais concretas e aprofundadas.

Para que os experimentos alcançassem uma maior eficácia, foram desenvolvidos os roteiros para a execução correta de cada equipamento, tanto da Feira quanto do Planetário. Além disso, os integrantes passaram por um treinamento específico com o intuito de ampliar o conhecimento teórico e prático acerca dos equipamentos.

Após o assentamento da atividade, o professor supervisor junto ao aluno coordenador do projeto Ciência em Órbita, faz-se a análise de alguns parâmetros relacionados a logística de transporte de equipamentos e pessoas, a disponibilidade de membros e ao tipo de linguagem usada durante a explicação. Estes parâmetros são: o local em que a atividade será desenvolvida, o público alvo da apresentação e os integrantes disponíveis para realizá-las. 


\section{Resultados e Discussão}

Executa-se os experimentos nas Feiras e Planetário Itinerantes com rodízios entre os integrantes da equipe PETFIS, o que proporciona visões e experiências diversificadas. Além disso, seguem-se os roteiros previamente estabelecidos com o passo a passo de cada equipamento, mas as abordagens adequam-se a cada faixa etária, de forma a proporcionar um ganho de conhecimento para cada geração.

Diante disso, a fim de contribuir com maiores conhecimentos e absorção dos conteúdos, agrupa-se os participantes em turmas distintas, conforme a quantidade e a faixa etária do público. Assim, durante as apresentações dos experimentos, direcionam-se perguntas para os participantes, com o intuito de despertar a atenção e curiosidade, além de estabelecer a interação com o público.

Na Figura 1, observa-se o Planetário Itinerante, o qual promove muita interação com o público durante as Feiras, e se mostra um importante instrumento de difusão de conhecimento. Isso está muito atrelado ao encanto que a Astronomia provoca nas pessoas.

Figura 1: Exposição do Planetário Itinerante na cidade Rio Piracicaba, MG. Em 1(a) equipe PETFIS com crianças antes da apresentação. Em 1 (b) crianças dentro do Planetário.

(a)

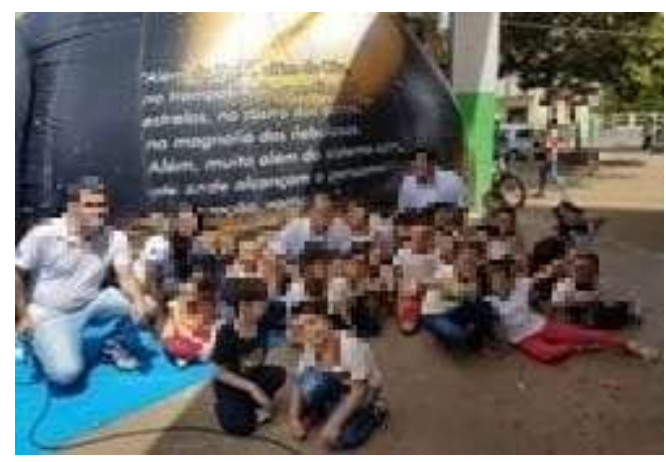

(b)

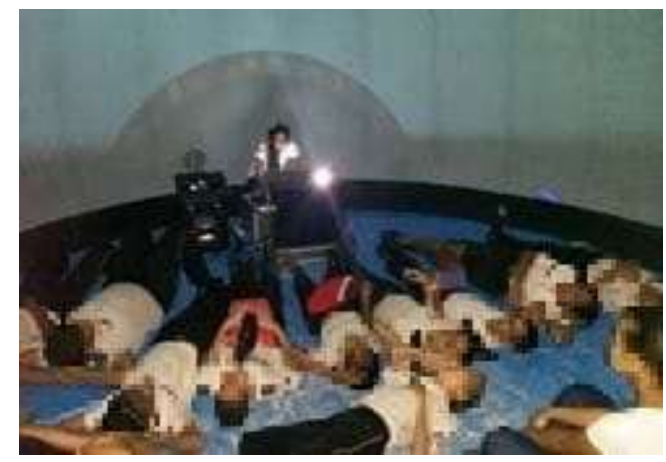

Fonte: Autores (2019).

O Planetário Itinerante é levado em eventos e escolas, e com sessões de, aproximadamente, 15 minutos, adaptadas à idade e maturidade do público-alvo, as crianças e adolescentes aprendem sobre o sistema solar, estrelas, galáxias e Astronomia de forma prazerosa e divertida. O Planetário é sempre a atração dos eventos.

Diversos equipamentos são utilizados pela equipe PETFIS no decorrer dos eventos. Todavia, alguns despertam maiores dúvidas e interesses por parte dos participantes. Sendo assim, em seguida mencionam-se os dois que mais atraíram a atenção: o gerador de Van de Graaff e a bobina de Tesla, conforme Figuras 2 e 3, respectivamente. Não obstante, o Globo de Plasma, demonstrado na Figura 3 (b), é um experimento que desperta bastante atenção do público participante, pois aborda o eletromagnetismo. Basicamente, se trata de uma bobina de Tesla dentro de globo com gás. 
Figura 2: Experimento com gerador de Van de Graaff. Em 2(a) Interação dos alunos observando centelha por meio do rompimento da rigidez dielétrica do ar, tornando-o condutor. Em 2(b) é mostrado o efeito de arrepiar o cabelo em uma boneca.

(a)

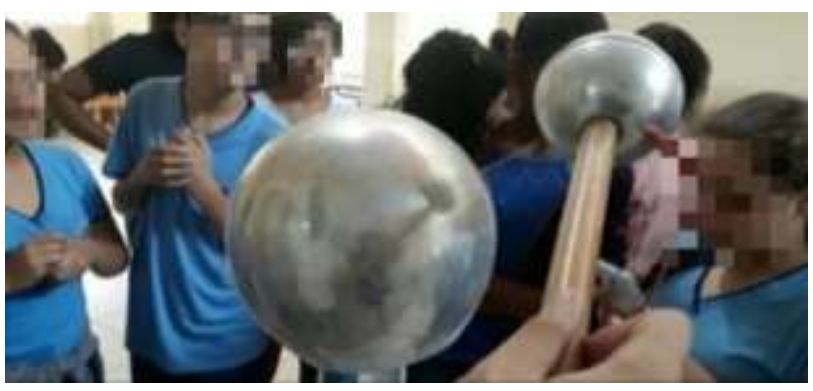

(b)

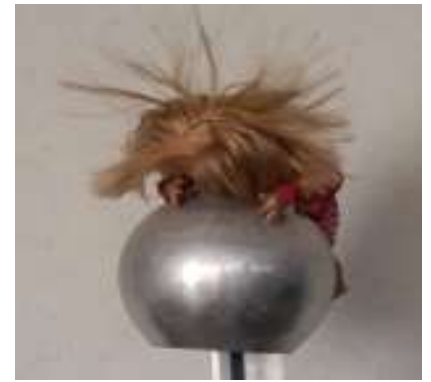

Fonte: Autores (2018).

Figura 3: Experimento com bobina de Tesla. Em 3(a), aluna acendendo uma lâmpada queimada por meio da d.d.p. gerada pela bobina. Em 3(b), o mesmo efeito com a bobina dentro do globo de plasma.

(a)

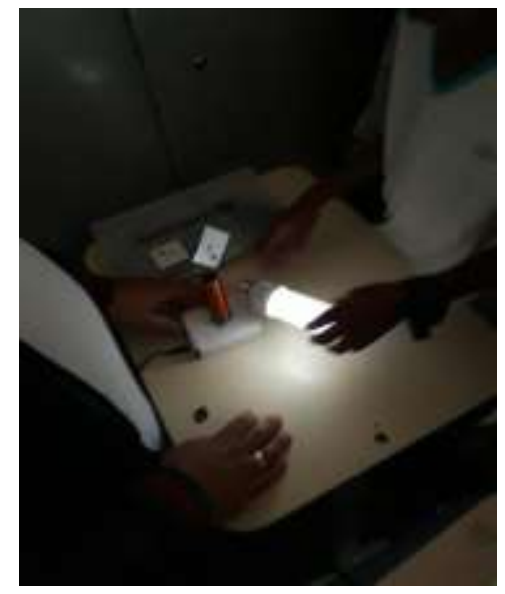

(b)

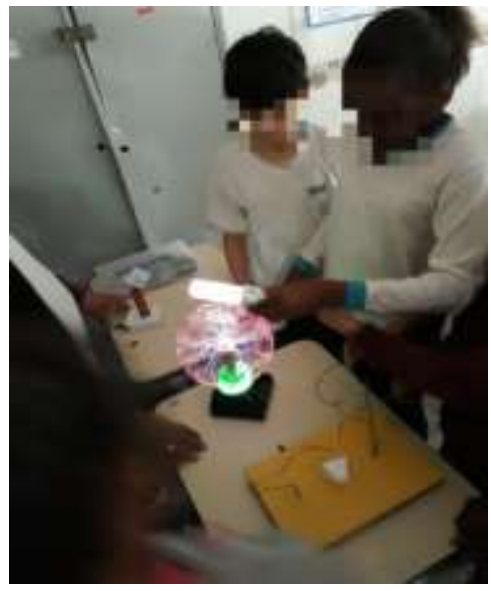

Fonte: Autores (2019).

Embora ambos os experimentos envolverem o mesmo tema - eletricidade - é notável que eles causam uma maior confusão por parte do público durante as apresentações, pelo fato de muitos pensarem que se tratam de um mesmo experimento. Consequentemente, grande parte intitula o gerador de Van de Graaff de bobina de Tesla, sendo que ambos as práticas são relacionadas com cargas elétricas, porém, o público não diferencia os processos de eletrização - Gerador de Van de Graff - e a indução por variação do fluxo magnético - Bobina de Tesla - que são os mecanismos de acúmulo de cargas. Além disso, outro ponto que leva a esta confusão, é pelo fato de "Tesla" estar mais difundido ultimamente na mídia.

Portanto, essas dúvidas são sanadas no decorrer da explicação. Ademais, as principais perguntas observadas sobre o gerador, por parte dos alunos, dizem respeito da causa da ocorrência da faísca e coloração, além de suas relações com o relâmpago e o trovão, bem como o arrepiar do cabelo e o grande receio de tomar choque.

Outro experimento que apresenta muita interação nas escolas, é a formação de bolhas de sabão gigantes, como mostra a Figura 4. Este é um experimento sempre solicitado pelas escolas que, permite trabalhar o conceito de tensão superficial. 
Figura 4: Formação de bolhas de sabão gigantes. Em 4(a), experimento cortina de bolha de sabão realizado com a participação do público. Em 4(b), o equipamento utilizado para esta prática.

(a)

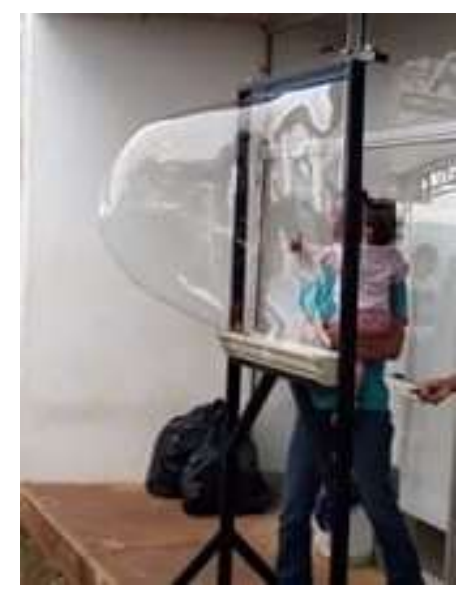

(b)

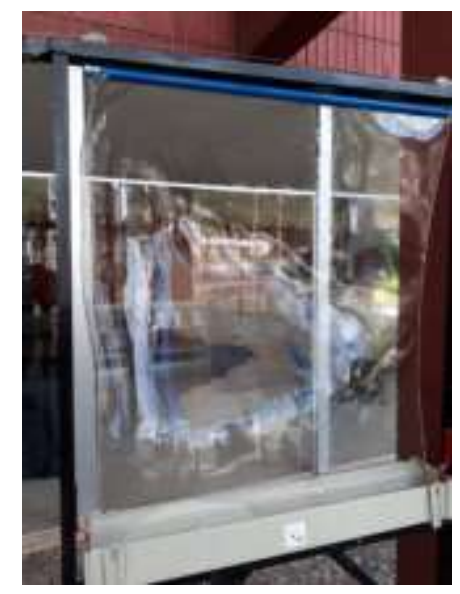

Fonte: Autores (2019).

Além disso, alguns professores de escolas em que os experimentos foram apresentados relataram grande motivação por parte dos alunos. Em algumas escolas, após a apresentação, os alunos construíram e interagiram com o grupo, por meio de um experimento usando uma bobina de um acendedor automático de um fogão para acender uma lâmpada queimada. Da mesma forma, as apresentações com o Planetário Itinerante também se mostraram efetivas para a mobilização dos alunos.

Mediante os dados apresentados, é de fundamental importância destacar que o projeto Ciência em Órbita promove um relevante impacto social, pois as atividades práticas contribuem para a assimilação da teoria exposta em sala de aula. O uso de Feiras e Planetário Itinerantes contribui na qualidade de método de ensino e aprendizado, no qual é categorizado como uma ferramenta eficaz e essencial para o desenvolvimento do conhecimento.

Ademais, salienta-se que em cada apresentação, é instantâneo e emocionante as reações das crianças e dos adolescentes. Os menores saem do Planetário eufóricos, enquanto os maiores saem deslumbrados com a possibilidade de começarem, mesmo de forma básica, a estudarem conceitos de Astronomia de maneira prazerosa.

Contudo, com as constantes apresentações, principalmente em relação ao Planetário Itinerante, desenvolveu-se um projeto em paralelo com o Colégio Municipal Professora Didi Andrade, em que se formou um grupo de estudos com o intuito de preparar os alunos para as Olimpíadas Brasileira de Astronomia e Astronáutica (OBA). Este projeto vem apresentando bons resultados, conforme desempenho e conquistas de medalhas (J. A. da Silva, Nepomuceno, \& Morais, 2019). É válido ressaltar que em 2020, os alunos participantes desta escola foram agraciados com medalhas de ouro, prata e bronze.

Torna-se claro, portanto, que é possível explicar com uma linguagem simples, lúdica e divertida experimentos que envolvem certa complexidade física para pessoas de diferentes faixas etárias e níveis de escolaridade, aproximando o ensino da ciência a diferentes realidades.

\section{Considerações Finais}

As Feiras e o Planetário Itinerantes geram impacto na forma de conhecimento, e dessa forma, percebe-se que o trabalho do PETFIS estabelece um retorno positivo à sociedade, ao âmbito científico e à universidade. Por conseguinte, o desenvolvimento destas atividades atraem mais integrantes para o meio acadêmico. Além disto, o aprender por meio da 
experimentação tem se mostrado cada vez mais eficaz, tanto para os integrantes da equipe, quanto para os alunos das escolas e eventos envolvidos com estes projetos.

Logo, uma das principais contribuições deste projeto é tornar a ciência mais agradável, de forma lúdica e prática, e, até mesmo, por meio de interação e visualização de fenômenos, esclarecer pontos que não são facilmente compreendidos em sala de aula. Ressalta-se, inclusive, o interesse dos participantes em entender as situações cotidianas.

Por meio destes projetos, é possível que os integrantes da equipe Conexão dos Saberes em Física e Popularização da Ciência trabalhem habilidades de oratória e interação social, habilidades estas fundamentais para sua vida pessoal e profissional. Além do aperfeiçoamento das técnicas de apresentação, conectam a Unifei e a sociedade, laço este que deve ser valorizado e reforçado. Ademais, para os que desejam seguir carreira acadêmica, os integrantes podem aperfeiçoar a habilidade de ensinar e transmitir conhecimento.

A interação do grupo com a população de Itabira e região é outro ponto fundamental para o desenvolvimento local por meio do incentivo à ciência. Desse modo, uma parte do conhecimento trabalhado e desenvolvido pela universidade aos integrantes é compartilhado à sociedade, principalmente aquela cuja participação ainda é nula, como forma de atrair mais integrantes e promover o senso crítico através do entretenimento e ensino de Física.

Ainda assim, as falhas percebidas pelos integrantes do PETFIS, em relação ao conhecimento dos participantes sobre os conteúdos abordados, no qual ocasionaram confusões de conceitos; abre, dessa forma, um viés para futuros estudos. É necessário identificar as causas destas falhas, que podem ser ocasionadas, como por exemplo, pelas diferenças entre o ensino público e privado, bem como a desigualdade social.

Portanto, é notável que as Feiras e o Planetário Itinerantes, geram significativas contribuições acerca dos integrantes e participantes dos eventos, uma vez que as atividades propiciam colaboração mútua entre os alunos que organizam o evento e o público participante. Contudo, ressalta-se a importância de adentrar as questões relacionadas às falhas nas bases de conhecimento, com estudos mais aprofundados sobre o assunto.

\section{Agradecimentos}

Os autores agradecem primeiramente a Deus, pela oportunidade e discernimento para a realização do projeto; ao Programa de Educação Tutorial/MEC pelo suporte financeiro e; a todos os colaboradores e participantes do programa Conexão de Saberes e Física e Popularização da Ciência da Unifei - Campus Avançado de Itabira.

\section{Referências}

Arantes, A. R. V., \& Barbosa, J. T. S. (2017). O lúdico na educação infantil. Revista Online de Magistro de Filosofia, Ano X(21), $100-115$.

Barcelos, N. N. S., Jacobucci, G. B., \& Jacobucci, D. F. C. (2010). Quando o cotidiano pede espaço na escola, o projeto da feira de ciências “"Vida em Sociedade"' se concretiza. Ciência \& Educação (Bauru), 16(1), 215-233. https://doi.org/10.1590/S1516-73132010000100013.

Costa, V. C. A., Alves, N. K. de O., Vasconcelos, F. S., Alvarenga, S. D., Geraldo, V., Yasuda, M. T., \& Morais, E. A. de. (2020). Uma forma lúdica de relacionar Poesia e Ciência. Research, Society and Development, 9(11), e8679119720. https://doi.org/10.33448/rsd-v9i11.9720

Everding, D. J., \& Keller, J. M. (2020). Survey of the academic use of planetariums for undergraduate education. Physical Review Physics Education Research, 16(2), 020128. https://doi.org/10.1103/PhysRevPhysEducRes.16.020128

Fernandes, B. M., Souza, A. R., Silva, R. P. da, Magalhães, J. P. P., Pereira, H. O., \& Morais, E. A. de. (2020). Contribuições do programa de educação tutorial: um estudo com egressos do grupo Conexão de Saberes em Física e Popularização da Ciência da Unifei-Itabira. Research, Society and Development, 9(11), e8919119642. https://doi.org/10.33448/rsd-v9i11.9642

Freitas, W. R. de S., \& Jabbour, C. J. C. (2010). O estudo de caso como estratégia de pesquisa qualitativa: Fundamentos, roteiro de aplicação e pressupostos de excelência. In XXX Encontro Nacional de Engenharia de Produção. São Carlos. http://www.abepro.org.br/biblioteca/enegep2010_tn_sto_122_790_15342.pdf

Germano, M. G., \& Kulesza, W. A. (2007). Popularização da ciência: uma revisão conceitual. Caderno Brasileiro de Ensino de Física, 24(1), 7-25. 
Germano, M. G., \& Kulesza, W. A. (2011). Uso das experiências de cátedra no ensino de Física. Caderno Brasileiro de Ensino de Física, 21(2), 145-175. Retrieved from https://periodicos.ufsc.br/index.php/fisica/article/view/1546/5617\%0Ahttps://periodicos.ufsc.br/index.php/fisica/article/view/6430/5946

Higa, I., \& Oliveira, O. B. de. (2012). A experimentação nas pesquisas sobre o ensino de Física: fundamentos epistemológicos e pedagógicos. Educar Em Revista, 44, 75-92.

Langhi, R., \& Nardi, R. (2009). Ensino da astronomia no Brasil: educação formal, informal, não formal e divulgação científica. Revista Brasileira de Ensino de Fisica, 31(4). https://doi.org/10.1590/s1806-11172009000400014

Lantz, E. (2011). Planetarium of the Future. Curator: The Museum Journal, 54(3), 293-312. https://doi.org/10.1111/j.2151-6952.2011.00093.x

Miguel, P. A. C. (2007). Estudo de caso na engenharia de produção: estruturação e recomendações para sua condução. Production, 17(1), 216-229. https://doi.org/10.1590/S0103-65132007000100015

Morais, E. A. de, Pedro, D. F. P., Fernandes, B. M., Silva, J. J. R., Cruz, M. P., Nogueira Neto, L. F., ... Geraldo, V. (2020). Curso Popular Preparatório para o Exame Nacional do Ensino Médio: um meio de Inclusão Social em Itabira. Research, Society and Development, 9(5), e60952849. https://doi.org/10.33448/rsdv9i5.2849

Oaigen, E. R., Bernard, T., \& Souza, C. A. (2013). Avaliação do Evento Feiras de Ciências: Aspectos Científicos, Educacionais, Socioculturais e Ambientas. Revista Destaques Acadêmicos, 84-94.

Pereira, E. B., \& Robaina, J. V. L. (2020). Estudo do conhecimento sobre Feira de Ciências nas Atas do ENPEC e na Base REDALYC: aspectos significativos ao processo de ensino e aprendizagem no Ensino Médio. Research, Society and Development, 9(7), e696974821. https://doi.org/10.33448/rsd-v9i7.4821

Silva, J. A. da, Nepomuceno, L. A., \& Morais, E. A. de. (2019). Oficinas de Astronomia - Preparação para OBA: Forma Lúdica de Aprendizagem. In Anais do IV CBEPF (p. 1). São José do Rio Preto.

Silva, M. F. da, \& Mendoza, C. C. G. (2020). A importância do ensino, pesquisa e extensão na formação do aluno do Ensino Superior. Revista Científica Multidisciplinar Núcleo Do Conhecimento,119-133. https://doi.org/10.32749/nucleodoconhecimento.com.br/educacao/pesquisa-e-extensao

Türk, C., \& Kalkan, H. (2015). The Effect of Planetariums on Teaching Specific Astronomy Concepts. Journal of Science Education and Technology, 24(1), 1-15. https://doi.org/10.1007/s10956-014-9516-6

Zhukova, G., \& Bukgakova, T. (2019). University Planetariums and Observatories: The Critical Role of Higher Education in Future Studies. Philosophy and Cosmology, 23. https://doi.org/10.29202/phil-cosm/23/12 